\title{
BMJ Global Health Stop 'controlling' for sex and gender in global health research
}

\author{
Janna R Shapiro (D) ,' Sabra L Klein, ${ }^{1,2}$ Rosemary Morgan ${ }^{1}$
}

To cite: Shapiro JR, Klein SL, Morgan R. Stop 'controlling' for sex and gender in global health research. BMJ Global Health 2021;6:e05714. doi:10.1136/ bmjgh-2021-005714

Handling editor Seye Abimbola

Received 15 March 2021 Revised 26 March 2021 Accepted 27 March 2021
Check for updates

(c) Author(s) (or their employer(s)) 2021. Re-use permitted under CC BY-NC. No commercial re-use. See rights and permissions. Published by BMJ.

${ }^{1}$ International Health, Johns Hopkins University Bloomberg School of Public Health, Baltimore, Maryland, USA ${ }^{2}$ Molecular Microbiology \& Immunology, Johns Hopkins University Bloomberg School of Public Health, Baltimore, Maryland, USA

Correspondence to Dr Rosemary Morgan; rosemary.morgan@jhu.edu
If you read any global health publicationwhether it be about injury prevention, noncommunicable diseases or vaccines-you are likely to find a footnote in a table or a sentence in the statistical methods section indicating that the results were 'controlled' for sex or gender. Although the terms sex and gender are often used interchangeably in the literature, the distinction between them is important. Sex, or the biological differences between males and females, is based on the sex chromosome complement, reproductive tissues and sex steroid concentrations. In contrast, gender is based on behaviours, occupations and activities defined by social or cultural norms, and can refer to differences among men, women and gender minorities. There is ample evidence that both sex and gender contribute meaningfully to global health outcomes. In this article, we explore what it means when we 'control' for sex or gender, and whether this practice can have unintended outcomes.

Statistically, we seek to uncover how predictors influence a health outcome. In global health, predictors can be demographic (eg, age or race), medical (eg, type or presence of treatment) or intervention based (eg, access to intervention or not). In many cases, a third type of variable, known as a 'confounder', must also be taken into account. In the statistical literature, there are many technical definitions of confounding. ${ }^{1}$ For our purposes, a confounding variable is a risk factor for the outcome that is also associated with the predictor, such that the observed relationship between predictor and outcome is confused by the presence of the confounder (figure 1A). ${ }^{2}$ For example, in studying the relationship between age and the likelihood of getting a COVID-19 vaccine, sex or gender could be considered confounders if, in your study population, women were older and more likely to get the vaccine than men. In this case, sex, gender, or both, might make

\section{Summary box}

Sex and gender are often 'controlled' for in global health research, which forces the relationship between the predictor and outcome of interest to be the same across sex (ie, males, females and intersex) or gender (ie, men, women and gender minorities).

- There are many examples where controlling for sex, gender or both led to incorrect findings that were detrimental to equitably improving global health.

- Instead of controlling for sex or gender, we urge researchers to consider sex and gender as variables of importance that can explain, rather than confound, their research.

it difficult to understand the causal relationship between age and the likelihood of vaccination.

Controlling for sex or gender means treating these variables as confounding factors, rather than variables of importance to the research question. Technically, this usually means that a term was included in a regression model to account for the fact that sex, gender, or both, might influence the predictor and the outcome, and possibly confuse the relationship under investigation. While this allows for sex or gender differences in the outcome at baseline, it also forces this difference to be the same at all levels of the predictor (note the parallel lines in figure 1A). For example, if we return to our example of how age (predictor) impacts the likelihood of getting the COVID-19 vaccine (outcome), controlling for sex or gender forces the difference between men and women to be the same at all ages. This approach assumes that the change in the likelihood of getting vaccinated with age is the same for men and women.

In reality, there are countless examples that demonstrate that the true relationships between our predictors and outcomes of interest do, in fact, differ by both sex and gender. We argue that relationships such as the ones depicted in figure 1B, where the sex/ 


\section{A. Controlling for sex/gender as confounding variables}
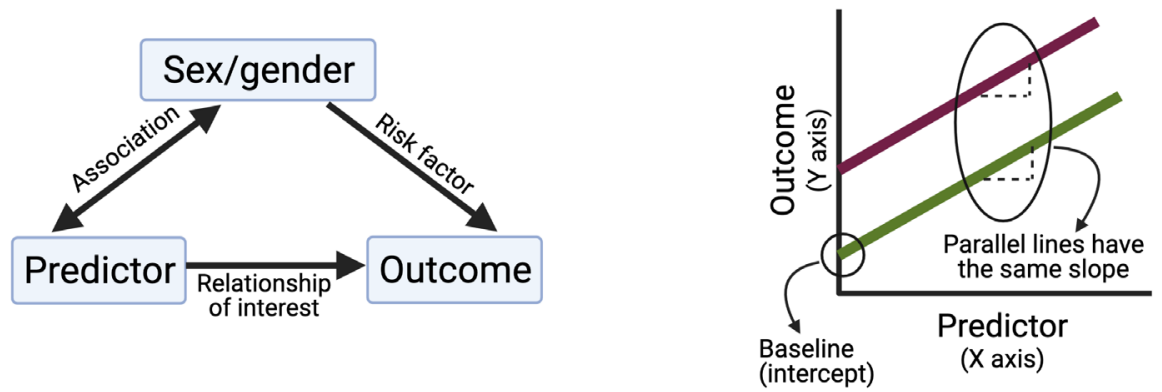

Sex/gender difference is constant at all levels of the predictor

\section{B. Including sex/gender as intersectional variables of interest}
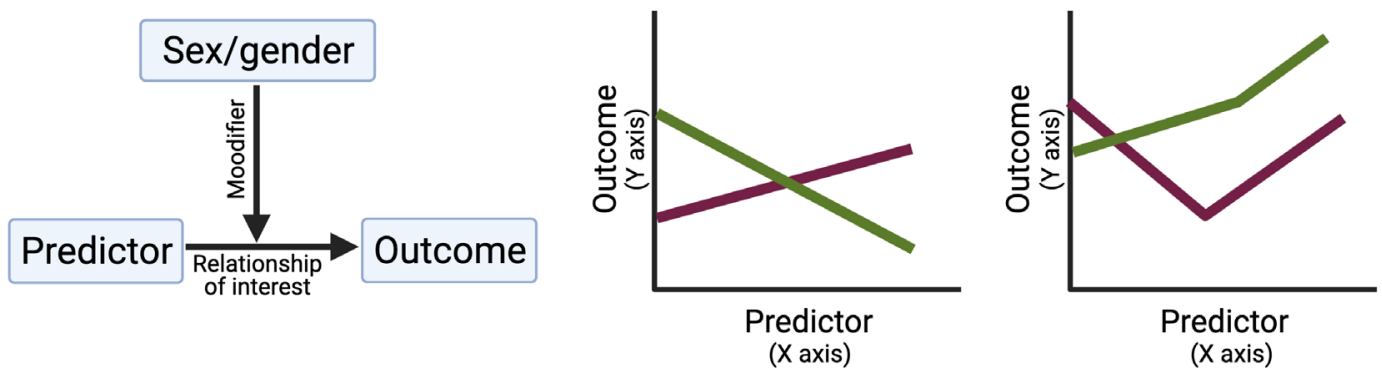
Sex/gender difference changes across
levels of the predictor

\section{Females/women $\square$ Males/men}

Figure 1 Controlling for sex and gender as confounding variables compared with including sex and gender as intersectional variables of interest.

gender difference changes across levels of the predictor, are often more accurate than the one depicted in figure 1A. There is considerable danger in ignoring such sex and gender differences by controlling for them statistically. For example, reanalysis of a randomised controlled trial comparing different antiretroviral therapy regimens for HIV management found higher rates of efficacy, adverse events and treatment discontinuation in women compared with men. ${ }^{3}$ Evidence suggests that this may be due to sex differences in drug metabolism, leading to higher drug exposure in females, ${ }^{2}$ yet the original analysis of this data completely ignored the role of sex as a biological variable by controlling for sex in statistical analyses. ${ }^{45}$ In this case, and in many others, assuming the outcome of the drug regimen was the same in men and women was not only incorrect, but detrimental to the health of women who were likely over-dosed, more likely to suffer side effects, and to discontinue treatment than men.

Although the tendency to 'control' for sex or gender is based in statistics, it is also pervasive in public health interventions and messaging, which often ignore sex as a biological variable or are blind to gender inequalities. For example, the link between gender norms and tobacco use has been thoroughly exploited by the tobacco industry. Tobacco advertising highlights associations between smoking and masculinity, or alternately, promotes smoking as a symbol of independence and sexuality to target women. ${ }^{6}$ Public health interventions for tobacco control, however, such as increasing prices and taxation of tobacco products, often do not consider the gendered aspect of this issue. ${ }^{6}$ The absence of gender-responsive tobacco control measures may explain why more countries saw significant decreases in the prevalence of smoking in men than in women between 2005 and 2015. ${ }^{7}$ Once again, treating sex and gender as confounding variables to be controlled for (or ignored), instead of meaningful sources of variation in the population, is detrimental to equitably improving global health.

Instead of controlling for sex and gender-be it statistically or in the application of an interventionwe urge those working in global health to consider sex and gender as variables of importance that can explain, rather than confuse, their research. A first, and necessary, step is to disaggregate data to interrogate how sex and gender intersect with each other or with the predictors and outcomes under investigation. ${ }^{8}$ Disaggregation of 
data is a trigger for sex-responsive and gender-responsive research that allows for understanding how the true relationship between a predictor and outcome differs between males and females or among men, women and gender minorities. This avoids the pitfalls and unintended consequences of ignoring sex as a biological variable and gender as a social variable, and adds richness and depth to the field of global health, which undoubtedly benefits the populations we serve.

Twitter Janna R Shapiro @JannaShapiro

Contributors RM conceptualised the manuscript. JRS wrote the manuscript, with guidance and editorial support from RM and SLK.

Funding This study was funded by National Institute on Aging U54AG062333 and the National Cancer Institute U54CA260492

Competing interests None declared.

Patient consent for publication Not required.

Provenance and peer review Not commissioned; externally peer reviewed.

Data availability statement There are no data in this work.

Open access This is an open access article distributed in accordance with the Creative Commons Attribution Non Commercial (CC BY-NC 4.0) license, which permits others to distribute, remix, adapt, build upon this work non-commercially, and license their derivative works on different terms, provided the original work is properly cited, appropriate credit is given, any changes made indicated, and the use is non-commercial. See: http://creativecommons.org/licenses/by-nc/4.0/.
ORCID iD

Janna R Shapiro http://orcid.org/0000-0001-8088-2506

\section{REFERENCES}

1 VanderWeele TJ, Shpitser I. On the definition of a confounder. Ann Stat 2013:41:196

2 Gordis L. More on causal inferences: bias, confounding, and interaction. Epidemiology 2000;4:247-64.

3 Squires KE, Johnson M, Yang R, et al. Comparative gender analysis of the efficacy and safety of atazanavir/ritonavir and lopinavir/ ritonavir at 96 weeks in the Castle study. J Antimicrob Chemother 2011;66:363-70

4 Ofotokun I, Chuck SK, Hitti JE. Antiretroviral pharmacokinetic profile: a review of sex differences. Gend Med 2007:4:106-19.

5 Molina J-M, Andrade-Villanueva J, Echevarria J, et al. Once-Daily atazanavir/ritonavir compared with twice-daily lopinavir/ritonavir, each in combination with tenofovir and emtricitabine, for management of antiretroviral-naive HIV-1-infected patients: 96-week efficacy and safety results of the Castle study. J Acquir Immune Defic Syndr 2010;53:323-32.

6 Gender-responsive tobacco control. Evidence and options for policies and programmes. Geneva, Switzerland: Secretariat of the WHO Framework Convention on Tobacco Control; World Health Organization, 2018.

7 GBD 2015 Tobacco Collaborators. Smoking prevalence and attributable disease burden in 195 countries and territories, 19902015: a systematic analysis from the global burden of disease study 2015. Lancet 2017;389:1885-906.

8 Shapiro JR, Klein SL, Morgan R. COVID-19: use intersectional analyses to close gaps in outcomes and vaccination. Nature 2021;591:202. 